\title{
A note from Patrick J. Cozzone, MAGMA Editor-in-Chief
}

\author{
Patrick J. Cozzone ${ }^{1,2}$
}

Published online: 17 November 2017

(c) ESMRMB 2017

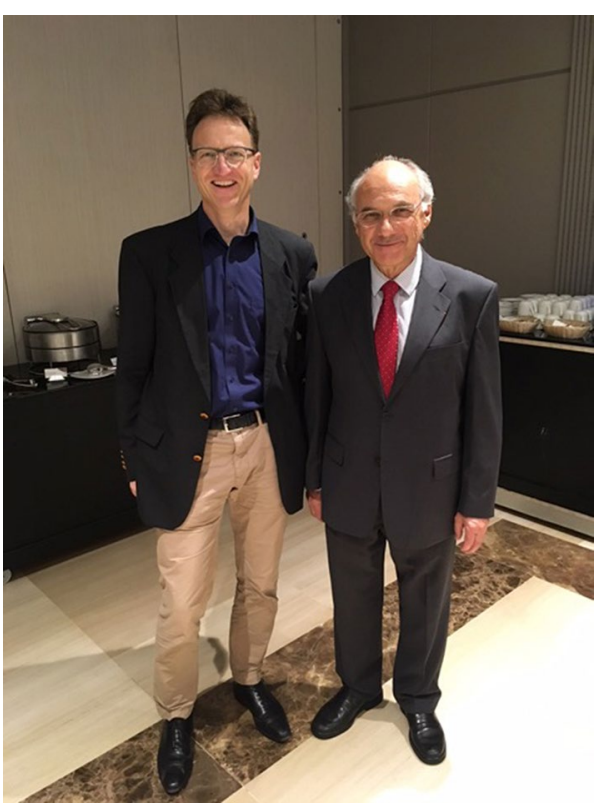

Prof Norris and Prof Cozzone, Barcelona, October 2017

Fifteen years already! Yes, it has been my privilege to serve the magnetic resonance (MR) community for 15 years as Editor-in-Chief of Magnetic Resonance Materials in Physics, Biology and Medicine (MAGMA), succeeding in 2003 Axel Haase, the first Editor-in-Chief of the journal. After all these years and this long, challenging, and enjoyable journey, I felt the time had come to step down and allow new blood to come in and take the journal to a new stage of development and recognition. The ad hoc Search Committee

Patrick J. Cozzone

patrick_cozzone@sbic.a-star.edu.sg

1 Singapore Bioimaging Consortium, 11 Biopolis Way, \#02-02 Helios, Singapore 138667, Singapore

2 Centre de Résonance Magnétique Biologique et Médicale, Faculté de Médecine, Aix-Marseille Université, 27 Boulevard Jean Moulin, 13005 Marseille, France set up by the European Society for Magnetic Resonance in Medicine and Biology (ESMRMB), after conducting a worldwide search, unanimously voted to appoint David Norris, a proposition readily confirmed by the General Assembly of ESMRMB held in Barcelona on Saturday, October 21, 2017. David Norris has profound experience in editing scientific journals, he is well recognized and respected internationally in our MR community, he masters many aspects of MR research and development, and he is already familiar with MAGMA (he has been an active member of the MAGMA Editorial Board for almost two decades). He will take over his new responsibilities with the first issue of 2018.

Since 2003, MAGMA has gone through many stages of development, always accompanied and supported by Springer, our publisher, and ESMRMB. Electronic online submission was introduced in 2005, first with Manuscript Central, then moved to Editorial Manager in 2014. Open access publications were made available very early on. Adhering to the highest standards in publication ethics has always been a priority for the MAGMA Editorial Team, and since 2012 the journal has strictly followed the Code of Conduct and Best Practice Guidelines for Journal Editors of the Committee on Publication Ethics (COPE) promoting integrity in research and its publication. In this context, I have been vigilant in avoiding all manipulations of the impact factor, whatever one might think about the limitations of this metric as a token of a journal's quality. Over the years, international recognition of MAGMA has increased steadily. The growth in the number of articles submitted has led to an increase of the page budget, each issue containing now a minimum of 100 pages. MAGMA is widely disseminated worldwide, mostly through Springer library consortia, reaching close to 9000 scientific institutions in 2017. Downloads of the full text of MAGMA articles have grown exponentially, currently reaching an average of 200 downloads per day! The journal has moved from print to an electronic version, and from 2018, it will be available in an electronic version only. In addition to original peer-reviewed research articles, MAGMA has published to date 11 successful special issues on hot topics that have been particularly 
well received by the MR community as judged by the high number of citations they have generated, even several years after their initial publication.

I would like to thank current and former Editorial Board members for their unwavering support and commitment to the standards to which MAGMA aspires. In addition, I am grateful to our pool of about 2000 international reviewers. Their diligence has been a major contribution to the very competitive timelines, both from submission to review and from acceptance to publication, that MAGMA offers to authors, probably the shortest among all MR journals.

The successful development of MAGMA has derived largely from the hard work, expertise, and dedication of Marguerite Izquierdo, my editorial assistant. She has had few breaks to relax from journal activities since 2003, and she is probably ready to take a long and well-deserved vacation when she also steps down at the end of 2017.
The change in leadership at MAGMA marks the beginning of a new stage of development. I have no doubt that David Norris, as the new Editor-in-Chief, is fully qualified to take MAGMA to the next level and to position it firmly within the top journals in MR.

Patrick J. Cozzone

MAGMA Editor-in-Chief 2003-2017

November 2017

\section{Compliance with ethical standards}

Conflict of interest The author declare that they have no competing interests

Ethical standards This editorial does not contain any studies with human participants or animals 